\title{
MOLECULAR DESIGN OF POLYCYCLIC AROMATIC HYDROCARBONS BASED ON PRECISE PPP MOLECULAR ORBITAL CALCULATIONS
}

\author{
KIMIHIRO HIRUTA ${ }^{\mathrm{a}}$, SUMIO TOKITA ${ }^{*}$, YOSHIHIRO DAIKOKU ${ }^{\mathrm{a}}$, \\ and KICHISUKE NISHIMOTO ${ }^{b}$ \\ a Department of Applied Chemistry, Faculty of Engineering, Saitama University, \\ 255 Shimo-Ohkubo, Urawa, Saitama, 338 Japan \\ b Institute for Fundamental Chemistry, 34-4 Takano-Nishihiraki-cho, \\ Sakyo-ku, Kyoto 606, Japan
}

Applying the procedure of precise Pariser-Parr-Pople molecular orbital (PPP MO) calculations, in which a novel two center electron repulsion integral new- $\gamma$ was used and spectrochemical sof tness parameter in the new$\gamma$ was evaluated based on the spectroactive aromatic sextet resonance system (ASRS) of a molecule, the wavelengths of the $p$-band of acenes and their higher analogues were predicted. The relationship between the annellation and the shift of the wavelength of the $p$-band was revealed. The guideline to design PAHs which possess the long absorption wavelengths was proposed.

\section{Introduction}

The computer-assisted theoretical design of functional organic molecules has become possible along with the recent progress of computer science [1]. In various molecular orbital (MO) methods, non-empirical $a b$ initio MO calculations is most sophisticated. However, ab initio MO calculations of electronic spectra of large organic molecules are expensive and impractical.

On the contrary, semi-empirical MO calculations, in particular Pariser-Parr-Pople (PPP) MO calculations, have been considered to be more practical for the prediction of absorption maxima of organic colorants [2-4]. Nishimoto proposed a novel two center electron repulsion integral new- $\gamma$, which takes the spectrochemical softness into account, for the PPP MO calculations [5]. The new- $\gamma$ was successfully applied for the precise calculations of absorption wavelengths of the $p$-band, which especially affects the visual color, of polycyclic aromatic hydrocarbons (PAHs) [6-9]. The calculated results using new- $\gamma$ were more accurate than the ones using the conventional Nishimoto* Mataga $\gamma(\mathrm{N} \cdot \mathrm{M}-\gamma)$ function [10].

In this paper, we would like to report the relationship between the annellation of acenes (1) and their higher analogues (2-4) (Fig. 1) and the shift of the wavelength of the $p$-band of them by means of the precise PPP MO calculations. We propose the guideline of the molecular design of PAHs which possess the long absorption wavelengths. 


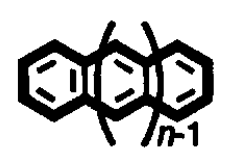

1

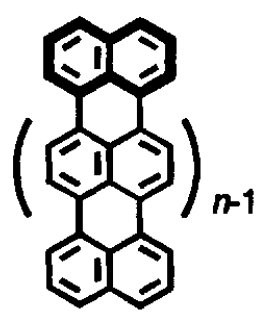

2

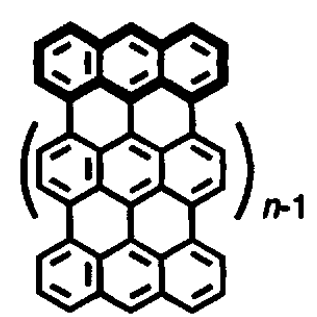

3

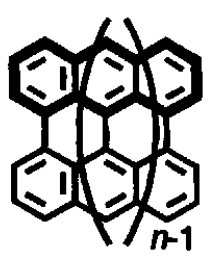

4

Fig. 1 Structural formulae; spectroactive ASRS is represented using bold lines

\section{Method}

PPP MO calculations were performed with a computer software PPP-PC $[4,11]$ in which variable $\beta$ approximation [12] and the conventional parameters set $[4,13]$ were used. For two center electron repulsion integral, either the new- $\gamma$ [5] or the $N \cdot M-\gamma$ [10] was used with 25 lower singly excited configurations in $\mathrm{Cl}$ calculations.

New- $\gamma$ is represented as

$$
\gamma=e^{2} /\left(R_{r s}+k a_{r s}\right)
$$

where $R_{r s}$ is the interatomic distance (in $A$ ) between the $r$-th and $s$-th atoms in a molecule containing $\pi$-electrons; $a_{r s}$ is given by

$$
a_{r s}=2 e^{2} /\left(I_{r}-A_{s}+I_{s}-A_{r}\right)
$$

where $e^{2}$ is $14.397 \mathrm{eV} \cdot \AA, I_{r}\left[I_{s}\right]$ and $A_{r}\left[A_{s}\right]$ are the valence state ionization potential and the electron affinity, respectively. Thus, $k$ is a dimensionless parameter which indicates the relative magnitude of mobile $\pi$-electron polarization at the region between $r$-th and $s$-th atoms, namely, the spectrochemical softness of $\pi$-electrons [5]. When the value of $k$ is 1 , new- $\gamma$ is equivalent to the $N$. M- $\gamma$.

\section{Results and Discussion}

Acenes (1) are the most fundamental series in PAHs, and the absorption wavelengths of the $p$ band of them shift toward longer wavelengths along with the annellation $[14,15]$. We defined acenes as aromatic sextet resonance system (ASRS) based on the essential character of their mobile $\pi$-electrons [7]. On the contrary, the absorption wavelengths of the $p$-band of zig-zag phenes (phenanthrene, chrysene, picene, and so on) shift toward longer wavelengths more slightly compared with those of acenes, along with the annellation. We defined zig-zag phenes as aromatic sextet alternation system (ASAS), because their $\pi$-electrons trend to localize at the particular positions of the molecular framework [7].

The parameter $k$ suitable for the calculations of the absorption wavelengths of the $p$-band of PAHs were evaluated based on the spectroactive ASRS of a molecule [9]. The spectroactive ASRS 
Table 1 Calculated and observed absorption wavelengths of the p-band of PAHs 1-4

\begin{tabular}{|c|c|c|c|c|c|}
\hline \multirow{2}{*}{ Compound } & \multirow{2}{*}{$n$} & \multirow{2}{*}{$\lambda_{\text {obs. }}{ }^{\mathrm{a}}$} & \multicolumn{3}{|c|}{$\lambda_{\text {calc. }}$} \\
\hline & & & $N \cdot M-\gamma^{b}$ & new- $\gamma^{c}$ & Clar ${ }^{d}$ \\
\hline \multirow{10}{*}{1} & 0 & 208 & 201 & 211 & 205 \\
\hline & 1 & 283 & 281 & 282 & 279 \\
\hline & 2 & 367 & 356 & 373 & 364 \\
\hline & 3 & 458 & 420 & 461 & 461 \\
\hline & 4 & 556 & 481 & 556 & 569 \\
\hline & 5 & 653 & 534 & 649 & 689 \\
\hline & 6 & & 581 & 756 & 820 \\
\hline & 7 & & 619 & 848 & 962 \\
\hline & 8 & & 651 & 939 & 1116 \\
\hline & 9 & & 678 & 1023 & 1281 \\
\hline \multirow{5}{*}{2} & 0 & 283 & 281 & 282 & \\
\hline & 1 & 417 & 407 & 413 & \\
\hline & 2 & 524 & 501 & 513 & \\
\hline & 3 & 611 & 579 & 596 & \\
\hline & 4 & & 642 & 663 & \\
\hline \multirow{4}{*}{3} & 0 & 367 & 356 & 373 & \\
\hline & 1 & 614 & 566 & 608 & \\
\hline & 2 & & 769 & 837 & \\
\hline & 3 & & 972 & 1056 & \\
\hline \multirow{5}{*}{4} & 0 & 244 & 258 & 260 & \\
\hline & 1 & 417 & 407 & 413 & \\
\hline & 2 & 614 & 566 & 608 & \\
\hline & 3 & & 711 & 817 & \\
\hline & 4 & & 840 & 1010 & \\
\hline
\end{tabular}

${ }^{a}$ Extrapolated values to the gas phase (references 14 and 16). ${ }^{b}$ Conventional Nishimoto $\cdot$ Mataga $\gamma$ function. ${ }^{c}$ New- $\gamma$ based on the spectroactive ASRS. ${ }^{d}$ Prediction using Clar's expression (reference 14).

is a portion which contributes mainly to the electronic spectra of PAHs. We defined the number of hexagons included in the spectroactive ASRS of PAHs as a parameter $l$. The regression expression (3) to evaluate the spectrochemical softness parameter $k$ was shown as follows [9]:

$$
k=0.33 l+0.48
$$

The spectroactive ASRSs of PAHs 1-4 are represented using bold lines in Fig. 1, and parameter $k$ for them are evaluated using expression (3).

The calculated wavelengths in the gas phase of the $p$-band of acenes (1) using the new- $\gamma$ or the $\mathrm{N} \cdot \mathrm{M}-\gamma$ are shown in Table 1. Clar proposed the empirical expression (4) to predict wavelengths of 1 as follows [14]: 
Table 2 The statistical parameters for the linear relationship between the number of $\pi$-electrons and the absorption wavelengths of the $p$-band of PAHs 1-4 $(\lambda=a \cdot P E+b ; r$ is a correlation coefficient)

\begin{tabular}{ccccc}
\hline Compound & $n$ & $a$ & $b$ & $r$ \\
\hline 1 & $0 \sim 9$ & 23.1 & 55.2 & 0.9995 \\
2 & $0 \sim 4$ & 9.5 & 209.9 & 0.9916 \\
3 & $0 \sim 3$ & 16.3 & 149.0 & 0.9999 \\
4 & $0 \sim 4$ & 23.8 & -44.8 & 0.9987 \\
\hline
\end{tabular}

$$
\lambda_{p}=D^{2} / 16 R
$$

where $D$ is the number of reciprocal nuclear charges, and $R$ is the Rydberg constant. The calculated wavelengths using expression (4) for pentacene and hexacene (1,n=4 and 5) are longer than the observed ones (Table 1), so the predicted values for long acenes may be overestimated. On the other hand, the calculated wavelengths using the $N \cdot M-\gamma$ are shorter than the observed ones. The calculated wavelength using new $-\gamma$ may be more reasonable than other predicted ones, judging from the curve depicted in Fig. 2.

The wavelengths of annellated acenes derived formally by connecting all the peri-positions of acenes are calculated. The first member of them is "rylene" [14] series (2) which are annellated analogues of naphthalene $(1, n=1)$. The spectroactive ASRS of perylene $(2, n=1)$ is naphthalene unit, so the spectroactive ASRSs of other member of rylene series may be also naphthalene unit $(l=2, k=1.14)$. In fact, the calculated wavelengths of the $p$-band of $2(n=0-3)$ using new- $\gamma$ in which the parameter $k$ is evaluated as 1.14 reproduce well the observed ones (Table 1). Similarly, the spectroactive ASRSs of "anthene" [14] series (3) are regarded as anthracene unit $(l=3, k=1.47)$. The calculated wavelengths of the $p$-band of $3(n=0-3)$ are shown in Table 1 . The wavelengths of the $p$-band of $4(n=0-4)$, which are regarded as higher analogues of biphenyl and contains perylene $(2, n=1)$ and bisanthene $(3, n=1)$, are calculated (Table 1) to compare with those of 2 and 3.

The relationships between the annellation and the shift of the $p$-band of 1-4 are shown in Fig. 3. To evaluate them quantitatively, the statistical parameters for the linear relationship between the calculated wavelengths and the number of $\pi$-electrons $(P E)$ of 1-4 are used (Table 2): $\lambda_{p}=a \cdot P E+$ $b$, where $a$ is a slope, $b$ is a intercept, and $r$ is a correlation coefficient of this regression expression, respectively. For 2 and 3 , the values of $a$ are lower than that of 1 . Thus, the extension of $\pi$ conjugated system in $\mathbf{2}$ or $\mathbf{3}$ poorly contributes to the shift toward longer wavelength of the $p$-band, compared with that of 1 . In other words, the annellation from 1 to 2 or 3 poorly contributes to the extension of $\pi$-conjugated system. It is supported that the calculated bond order in the ground state of the bonds which connected two naphthalene units of perylene $(2, n=1)$ using PPP MO calculations is 0.33 , which is lower than that of other bond (Fig. 4). Thus, the character of the bonds which connected two naphthalene units is close to that of single bond. 


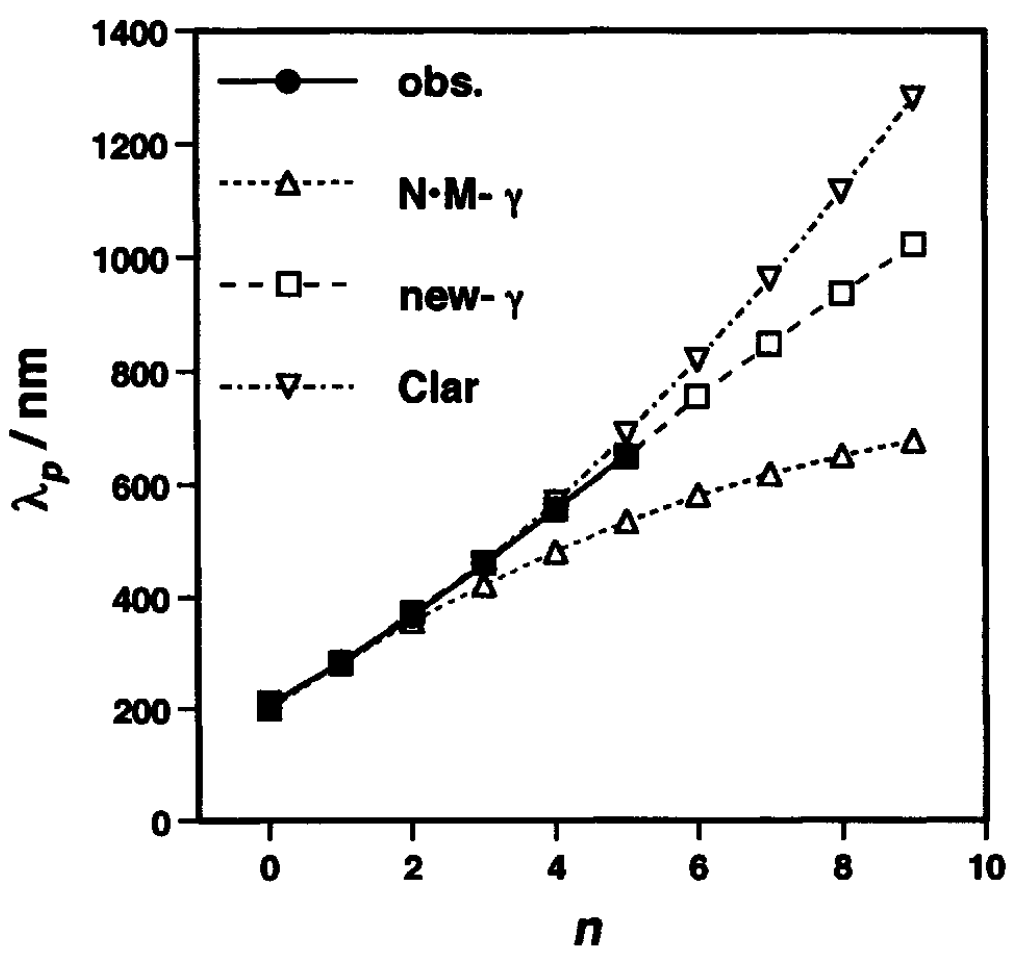

Fig. 2 The number $n$ of acenes (1) vs. the observed and calculated wavelengths of the $p$-band

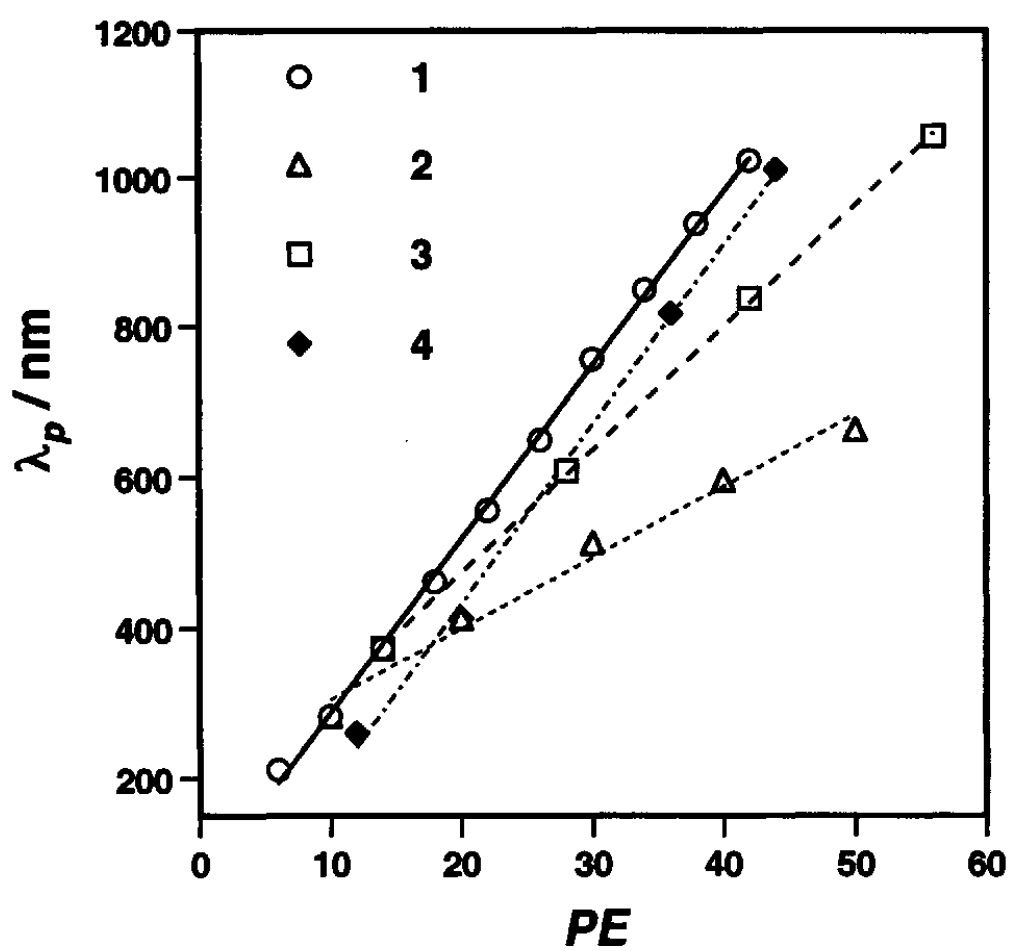

Fig. 3 The number of $\pi$ electrons $P E$ of 1-4 vs. the calculated wavelengths of the $p$-band 
On the other hand, the extension of $\pi$ conjugated system in 4 contributes to the shift toward longer wavelength of the $p$-band likewise that in 1. The value of $a$ of 4 is not two times of that of 1 , but almost identical with that of 1 . Thus, the annellation manner in 1 seems to be most effective to obtain PAHs which possess the long absorption wavelengths.

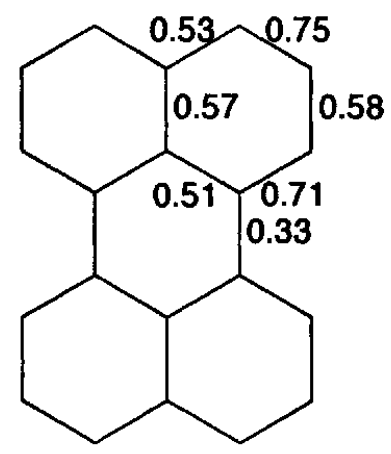

Fig. 4 Bond order in the ground state

Large PAHs are in general no easily accessible because of their high reactivity and poor solubility in common organic solvents. Koch et.al. [17] synthesized the four tert-butyl substituted derivative in the $\beta$-position of $2(n=4)$ which is sufficiently soluble in organic solvent to investigate their photophysical properties. Utilizing this method, some novel molecular frameworks described in this paper may be synthesized.

\section{References}

1. K. Nishimoto and A. Imamura Ed., Quantum Mechanics for the Molecular Design, Kodansha Scientific, Tokyo (1989).

2. J. Griffiths, Colour and Constitution of Organic Molecules, Academic Press, London (1976).

3. J. Fabian and H. Hartmann, Light Absorption of Organic Colorants, Springer-Verlag, Berlin (1980).

4. S. Tokita, M. Matsuoka, Y. Kogo and H. Kihara, Molecular Design of Functional Dyes -PPP Molecular Orbital Method and Its Applications, Maruzen, Tokyo (1989).

5. K. Nishimoto, Bull. Chem. Soc. Jpn., 66 (1993) 1876.

6. K. Hiruta, S. Tokita, Y. Kogo, H. Kihara and K. Nishimoto, J. Chem. Software, 1 (1993) 157.

7. K. Hiruta, S. Tokita and K. Nishimoto, J. Chem. Soc., Perkin Trans. 2, (1995) 1443.

8. S. Tokita, K. Hiruta and K. Nishimoto, Macromol. Symposia, in press.

9. K. Hiruta, S. Tokita and K. Nishimoto, Dyes and Pigments, in press.

10. K. Nishimoto and N. Mataga, Z. Physik. Chem. (Neue Folge), 12 (1957) 335.

11. F. Noguchi, S. Tokita, H. Kihara and K. Nishimoto, 3rd International Symposium of Functional Dyes, P37, Santa Cruz, California (1995).

12. K. Nishimoto and L. S. Forster, Theoret. Chim. Acta (Berl.), 3 (1965) 407.

13. K. Nishimoto and L. S. Forster, Theoret. Chim. Acta (Berl.), 4 (1966) 155; K. Nishimoto, Theoret. Chim. Acta (Berl.), 7 (1967) 207.

14. E. Clar, Polycyclic Hydrocarbons, Academic Press, London, (1964).

15. E. Clar, The Aromatic Sextet, Academic Press, London (1972).

16. D. Biermann and W. Schmidt, J. Am. Chem. Soc., 102 (1980) 3163, 3173.

17. K-H. Koch and K. Müllen, Chem. Ber., 124 (1991) 2091. 